gelkulturen erst 3 Tage nach Zugabe von $\mathrm{KNO}_{3}$ gestiegen war (Abb. 5), ist es wahrscheinlich, daß, ähnlich wie Pirson ${ }^{6}$ sowie Pirson, Tichy und Wilhelmi vermuten, die Regeneration von $\mathrm{N}$ Mangel-Zellen zunächst eine plasmatische ist, nach

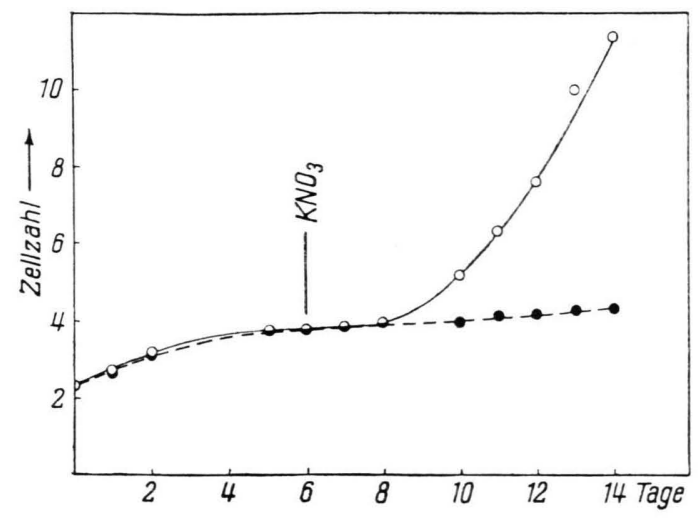

Abb. 5. Zellvermehrung einer N-Mangelkultur und einer solchen, die nach 6 Tagen $\mathrm{KNO}_{3}$ erhielt.

der dann eine Neubildung von Chlorophyll einsetzt, durch die eine weitere Photosynthese-Steigerung ermöglicht wird.

6 A. Pirs o n, Z. Bot. 31, 193 [1937].

7 A. Pirson, C. Tichy u. G. Wilhelmi, Planta 40, 199 [1952].
Bei den gelbgrünen Tomaten war nach den geschilderten Versuchen, durch Veränderung des K-NVerhältnisses in der Nährlösung einmal durch $\mathrm{K}$ Mangel eine normale Chlorophyllbildung zu erzielen, zum andern bei $\mathrm{K}$-Überschuß ein Ausbleichen der Blätter verursacht, wohl durch verringerte Chlorophyllbildung, aber auch durch Lichtschädigung. Das Verhältnis von Chlorophyll a und Chlorophyll b wurde dadurch nicht wesentlich beeinflußt. Es ist nach den vorliegenden Untersuchungen jedoch noch nicht klar, auf welchem Wege die Nährsalzwirkung geschieht. Ein veränderten K-Haushalt zieht im allgemeinen eine Fülle von Stoffwechselveränderungen nach sich. Vielleicht wirkt die abgewandelte $\mathrm{K}-\mathrm{N}$ Ernährung nur indirekt auf die eigentliche Ursache für die Störung der Lichtbeständigkeit der Chlorophyllführung. Weitere Versuche zu diesem Problem sind im Gange. Vor allem soll auch die Kombination mit anderen Nährsalzen, wie z. B. Phosphat, Mangan u. a., geprüft werden. Ferner soll untersucht werden, ob andere goldgelbe Pflanzen sich ähnlich verhalten. Hier soll vor allem festgehalten werden, daß diese Aureaform in ihrer Lichtempfindlichkeit beeinflußt werden konnte.

Ich danke Fräulein K ralik für die Mithilfe bei den Versuchen.

\title{
Die ontogenetische Entwicklung der Chloroplasten von Chlorophytum comosum
}

\section{Die Persistenz des Primärgranums und seine fragliche Kristallgitter-Struktur im Proplastid}

\author{
Von E. S. Perner
}

Aus dem Botanischen Institut der Westf. Wilhelmsuniversität Münster

(Z. Naturforschg. 11 b, 560-566 [1956]; eingegangen am 29. Juni 1956)

\begin{abstract}
Im Vegetationskegel, in den Blattanlagen und jungen Blättern von Chlorophytum comosum sind in Übereinstimmung zu lichtmikroskopischen (1.-m.) Befunden auch elektronenmikroskopisch (e.-m.) Proplastiden nachzuweisen, welche im Stroma mindestens ein stark elektronenstreuendes Primärgranum enthalten. Dieses wird aus ca. $190 \AA$ großen, kugeligen „Elementareinheiten “ aufgebaut, welche im Abstand von etwa $450 \AA$ linear auf fädigen Strukturen aufgereiht sind. Diese perlketten-artigen Fäden sind in bevorzugten Ebenen miteinander vernetzt und bilden so flächengitter-artige Lamellen. Sie sind so zu einem komplexen Schichtenkörper geordnet, daß das Primärgranum wie ein Kristall aussieht. Das Primärgranum ist kein Chlorophyllkristall, sondern muß vielmehr als persistierender Chromo-Nucleo-Proteid-Komplex betrachtet werden.
\end{abstract}

I $m$ meristematischen Blatt- und Sproßgewebe verschieneder höherer Pflanzen ist in den letzten Jahren durch eine Reihe von Untersuchern ${ }^{1}$ 1.- und e.-m. die Existenz von Proplastiden als primären Entwicklungstadien soma-

1 Vgl. S. Strug ger u. E. Perner, Protoplasma 46, 711 [1956], dort zusammenfassende Literatur.

tischer Chloroplasten sichergestellt worden, nachdem S trug g e r sie zum erstenmal bei Liliifloren auffinden konnte $2^{-4}$.

2 S. S trugger, Naturwissenschaften 37, 166 [1950].

3 S. S trugger, Ber. dtsch. bot. Ges. 66, 439 [1953].

4 S. S trugger, Protoplasma 43, 120 [1954]. 
Nach den Ergebnissen der 1.-m. Analyse an fixiertem und gefärbten Zellmaterial ergibt sich im Zusammenhang mit Lebendbeobachtungen die Feststellung, daß das Proplastid aus einem amöboid formveränderlichen „Stroma“ besteht, in welches ein persistierendes, nach Fixation stark färbbares „Primärgranum“ eingebettet ist. Schon im basalen Blattmeristem führen Proplastiden Chlorophyll, intra vitam besitzt aber nur das Primärgranum eine elektive, blutrote Chlorophyllfluoreszenz ${ }^{5}$. Stärkekörner lassen sich nur im Stroma feststellen. Die Teilung der Proplastiden ist intra vitam beobachtet worden, das Auftreten bi- und multigranulärer Proplastiden spricht für die Teilungsfähigkeit des Primärgranums $2^{-4}, 6^{-4}$.

In den bis heute vorliegenden e.-m. Untersuchungen herrscht dagegen in Bezug auf Feinstruktur und Aufbau der Proplastiden keine Übereinstimmung. Die verschiedenen Autoren kommen unter Vernachlässigung l.-m. Kontrollen der betreffenden Objekte zu Vorstellungen, welche den 1.-m. Erfahrungen widersprechen. Lediglich $\mathrm{S}$ trug g e r und P e r n e r haben bei einer vergleichenden 1.- und e.-m. Untersuchung des Sproß-Vegetationskegels und junger Blätter von Helianthus tuberosus nachweisen können, daß in Übereinstimmung zu Monokotylen auch bei einer dikotylen Pflanze Proplastiden - aus „Stroma“ und „Primärgranum“ bestehend - vorhanden sind ${ }^{1}$. Im e.-m. Bild tritt das Primärgranum als lamellierter Schichtenkörper in Erscheinung, der in ein granuläres Stromaplasma eingebettet ist. Die Lamellen des Primärgranums stehen mit den erst später im Verlauf der Ontogenese zum somatischen Chloroplasten im Stroma gebildeten Lamellensystemen in kausaler Beziehung 1, 9, 10 . E.-m. ergeben sich auch Hinweise für die nach l.-m. Befunden zu fordernde Teilungsfähigkeit des Primärgranums.

Heitz und Maly ${ }^{11}$ lehnen die Persistenz des Primärgranums im Sinne der erstmalig von $\mathrm{S}$ t r u g g e r 2,3 vertretenen Auffassung auf Grund fluoreszenzmikroskopischer Lebendbeobachtungen ab. Das Proplastid, dessen Existenz nicht bezweifelt wird, soll primär nur aus undifferenziertem Stroma bestehen. Das dann von $\mathrm{Heit}_{\mathrm{z}}{ }^{12}$ e.-m. festgestellte „Granum junger Chloroplasten“ tritt erst sekundär im Verlauf der Ontogenese zum Chloroplasten in Erscheinung. Nachdem es eine kristallartige Innenstruktur besitzt 9, 12, wird es als einfacher bzw. zusammengesetzter Chlorophyllkristall gedeutet.

Auch $\mathrm{M}$ üh let hal e r ${ }^{10}$ findet bei der e.-m. Untersuchung von Aspidistra Proplastiden. Sie bestehen aber im primären Zustand lediglich aus einem stark elektronenstreuenden Primärgranum, das Stroma fehlt. Dieses soll erst in einem späteren Entwicklungszustand in Erscheinung treten.

5 S. S t r u g g e r, Naturwissenschaften 41, 286 [1956], dort Angaben zu den widersprechenden Befunden von E. H e itz u. R. M a ly, Z. Naturforschg. 8 b, 243 [1953].

6 J. B ö i n g, Protoplasma 45, 55 [1955].

7 G. Grave, Protoplasma 44, 273 [1954].

8 U. F a s s e-Franzisket, Protoplasma 45, 194 [1955].

9 H. L e y o n, Exp. Cell Res. 7, 609 [1954].

$10 \mathrm{~K}$. M ̈̈ h le th a le r, Protoplasma 45, 264 [1955].
Ausschließlich auf Grund e.-m. Untersuchungen lehnt L e y on ${ }^{13}$ unter Berücksichtigung seiner früheren Arbeiten die Existenz von Proplastiden im Sinne der Kontinuitätslehre von $\mathrm{Schimper}$ und $\mathrm{M}$ e ye r ab. Er beobachtet ,light and dense bodies“ und stellt dann in späteren Entwicklungsphasen ein „dense core“ fest.

Die e.-m. Befunde der einzelnen Autoren zeigen demnach keine Übereinstimmung untereinander und stehen dazu bei Heitz, Leyon und Mühlethaler in Widerspruch zu den bekannten l.-m. Grundlagen. Dementsprechend gehen auch die Auffassungen über die biologische Natur der Proplastiden und des darin nachweisbaren Primärgranums weit auseinander. Bei der Bedeutung des Problems der Plastidenentwicklung erscheint daher eine erneute e.-m. Analyse der Proplastiden bei Monokotylen dringend notwendig, welche nach vorliegenden Erfahrungen eng an 1.-m. Analysen anzuschließen ist ${ }^{1}$. Von besonderem Interesse dürfte aber die Frage nach der Feinstruktur des Primärgranums bei Liliifloren sein, nachdem diese Pflanzengruppe bevorzugtes Objekt sowohl für l.- als auch für e.-m. Analysen gewesen ist.

In den eigenen Untersuchungen erstreckt sich die Analyse auf den Sproß-Vegetationskegel und die jüngsten Blattanlagen sowie auf junge 1 bis $3 \mathrm{~cm}$ lange, noch kaum gelblich-grüne Blättchen einer nicht panaschierten Chlorophytum-Art (Chlorophytum comosum). Die Versuchspflanzen haben $2^{1 / 2}$ Tage verdunkelt gestanden und zeigen dann nur in Ausnahmefällen Stärkekörner in den Plastiden der untersuchten Gewebe. Die Fixation der schonend freipräparierten Objekte wurde mit 1-proz. Osmiumsäure (mit Veronal-Acetatpuffer auf $p_{\mathrm{H}} 7,2$ eingestellt) bei Zimmertemperatur und bei $+2^{\circ} \mathrm{C}$ vorgenommen (Fixationsdauer 3 bis 12 Stdn., zu Beginn immer Vakuum-Infiltration der Fixationsflüssigkeit). Die Einbettung in das Methacrylatgemisch erfolgte nach den Angaben von Rhodin ${ }^{14}$ bzw. den im Anatomischen Institut, Karolingska Institutet in Stockholm, üblichen Methoden*. Für die Herstellung der Ultradünnschnitte stand ein LKB-Gerät nach $\mathrm{S}$ jöst rand zur Verfügung, für die e.-m. Analye anfänglich in Stockholm ein RCA Emu 2 c, später in Münster (Westf.) ein Siemens Elmiskop I a.

\section{Untersuchungen zur Persistenz des Primärgranums}

$\mathrm{Heitz}{ }^{12}$ kommt bei der Untersuchung des gleichen Objektes zu der Ansicht, daß das von ihm beobachtete „Granum junger Chloroplasten“ nicht persistierend sei. E.-m. Belege liegen nicht vor, denn es sind lediglich 1 bis $3 \mathrm{~cm}$ lange Blättchen, nicht da-

11 E. Heitz u. R. M a ly, Z. Naturforschg. 8 b, 243 [1953].

12 E. Heitz, Exp. Cell Res. 7, 606 [1954]

13 H. L e y o n, Svensk kem. Tidskr. 68, 70 [1956].

14 J. R h od in, Thesis, Stockholm 1954.

* Herrn Prosektor Dr. F. S j ö s tr a n d und seinen Mitarbeitern sei an dieser Stelle für die persönliche Einweisung in die Ultramikrotomtechnik und für viele Ratschläge herzlichst gedankt, ebenso der Ges. d. Förderer u. Freunde der Westf. Wilhelmsuniversität in Münster (Westf.), die mir den Aufenthalt in Stockholm ermöglichte. 
gegen der meristematische Vegetationskegel-Bereich und jüngere Blattanlagen zur e.-m. Analyse herangezogen worden. Ebenso fehlen e.-m. Belege für die Hypothese, nach der es im Proplastid bei einer Anreicherung von Chlorophyll im Stroma zu einer Kristallisation - zur Bildung eines Granums - komme.

Dem stehen nun die Ergebnisse 1.-m. Untersuchungen am gleichen Objekt bzw. an nahe verwandten Pflanzen gegenüber, nach denen regelmäßig in allen Proplastiden von Meristemzellen mindestens ein primäres Granum nachweisbar gewesen ist ${ }^{1}$. $\mathrm{Zu}$ dem gleichen Ergebnis führen auch die hier vorgelegten e.-m. Untersuchungen, wenn man sie auf den Vegetationskegel-Bereich und jüngere Blattanlagen ausdehnt. Um allen möglichen Einwänden entgegnen zu können, sind umfangreiche Analysen notwendig gewesen. Nicht in allen Schnittserien sind Proplastiden so ideal geschnitten, daß im Stroma - den l.-m. Grundlagen entsprechend - auch das Primärgranum getroffen wurde. Dies ist verständlich, wenn man berücksichtigt, daß die Schnitte mit etwa 100 bis $200 \AA$ relativ dünn sind, die Proplastiden nicht sehr zahlreich vorkommen und dazu regellos im Cytoplasma verteilt liegen und sich die Schnittrichtung nicht von vornherein festlegen läßt. Es bleibt dem Zufall überlassen, wie ein Proplastid getroffen wird. Nicht zu vergessen ist auch die Tatsache, daß das Primärgranum im Verhältnis zum ganzen Proplastid doch relativ klein ist. Die Wahrscheinlichkeit, ideale Schnitte zu erhalten, ist demnach gering. Aus diesen Gründen ergibt sich die Notwendigkeit, zur Beurteilung ein umfangreiches Untersuchungsmaterial heranzuziehen und sich in keinem Falle mit Einzelschnitten zu begnügen. Wie der Vergleich e.-m. Bilder ein und desselben Proplastids, das in verschiedenen Ebenen getroffen wurde, gezeigt hat, können Einzelbilder zu falschen Vorstellungen führen.

Bei voller Berücksichtigung der technischen Schwierigkeiten führt die e.-m. Analyse von Chlorophytum comosum zu der Feststellung, daß niemals Proplastiden aufzufinden waren, denen ein Primärgranum offensichtlich fehlte. Den l.-m. Befunden entsprechend muß demnach das Primärgranum ein persistierender Bestandteil des embryonalen Proplastids sein, nachdem sich keinerlei Hinweise für eine spontane Neubildung ergab. Das ideal getroffene Proplastid zeigt im relativ schwach elektronenstreuenden, hellen und granulär aufgebauten Stroma einen stärker elektronenstreuenden, dunklen Komplex. Er ist nach Lage im Proplastid, Größe und Form mit dem „Primärgranum“ 1.-m. Untersuchungen identisch.
Teilweise ist im Bild auch die doppelt konturierte Grenzschicht zu erkennen, welche das Proplastid vom umgebenden Cytoplasma abgrenzt (Abb. $1^{*}$ ).

Abb. $2 \mathrm{a}$ und $\mathrm{b}$ zeigen ein und dasselbe Proplastid - ebenfalls aus dem Vegetationskegel-Bereich wie Abb. 1 - in verschiedenen Schnittebenen. Es ist langgestreckt und in der Mitte hantelförmig eingeschnürt. Ein Primärgranum ist hier nicht ohne weiteres festzustellen. Oben bzw. links (bei Abb. 2 b) sind jedoch tubuliartige Lamellen getroffen, welche im übrigen Teil des Proplastids fehlen. Bei diesen Schnitten ist das Primärgranum nicht getroffen worden, die Lamellen lassen jedoch vermuten, daß ein Primärgranum in diesem polaren Bereich zu suchen ist. Neben dem Proplastid ist ein Chondriosom angeschnitten, in dem dünne tubuliartige Röhren sichtbar werden. Entgegen der Ansicht von L e y o n lassen sich demnach im Meristem Proplastiden und Chondriosomen durchaus unterscheiden, wenn man den e.-m. Aspekt vergleicht ${ }^{13}$.

Auch Abb. 3 a zeigt noch ein Proplastid aus dem Vegetationskegel-Bereich. Ein Vergleich mit dem hantelförmigen Proplastid in Abb. 2 läßt vermuten, daß dieses polar getroffen wurde, so daß hier ein ungünstiger Querschnitt vorliegt. Die im Inneren sichtbaren osmiophilen Tröpfchen sprechen dafür, daß hier tatsächlich ein Proplastid und nicht ein Sphärosom getroffen wurde. Nach L e y on, M ühl e th a l e r u. a. sind solche Tröpfchen sub-l.-m. Größenordnung nicht nur im Proplastid, sondern auch im ausgewachsenen Chloroplasten beobachtet worden ${ }^{10.13}$. Die bei stärkerer Primärvergrößerung (Abb. $3 \mathrm{~b})$ sichtbaren Tubuli sprechen im Zusammenhang mit der starken Osmiophilie dafür, daß hier ein Primärgranum getroffen sein dürfte. Im linken Teil ist das schwach elektronenstreuende Stroma gerade sichtbar.

Abb. 4 und Abb. 5 zeigen dann Proplastiden aus etwa $0,5 \mathrm{~cm}$ langen Blättchen. Das Primärgranum läßt sich in allen drei Proplastiden einwandfrei identifizieren. Im oberen Plastid (Abb. 4) ist das Primärgranum gerade angeschnitten worden. Weiter nach links liegt ein Stärkekorn. Im unteren Plastid (Abb. 4) ist das Primärgranum besser getroffen und läßt bei gutem Erhaltungszustand auch bereits eine hohe Ordnung erkennen. In dem stärkefreien Proplastid der Abb. 5 ist die Existenz des Primärgranums ohne weiteres zu belegen. Abb. 6 zeigt dann ein ideal getroffenes Proplastid aus einem etwa $1 \mathrm{~cm}$ langen Blättchen. Als stark elektronenstreuender Komplex

\footnotetext{
* Abb. 1-14 s. Tafel S. 564 a-c.
} 
hebt es sich deutlich von dem hellen Stroma ab und zeigt nun die hohe Ordnung, welche $\mathrm{Heitz}$ als „Kristallgitter“ bezeichnet hat.

Nach diesen Befunden kann kein Zweifel bestehen, daß in allen Proplastiden des VegetationskegelBereichs, der jüngsten Blattanlagen und Blättern bis $\mathrm{zu} 1 \mathrm{~cm}$ Länge immer ein stark elektronenstreuender Komplex nachweisbar ist, der ein „Primärgranum“ darstellt. Betrachtet man die Größenverhältnisse der Proplastiden im Verlauf der Differenzierung der Blättchen aus dem Vegetationskegel, so ist eine GröBenzunahme des Proplastids und des Primärgranums festzustellen (vgl. 1. c. ${ }^{1}$ ). Die Länge der anfänglich amöboid formveränderlichen Proplastiden variiert zwischen 2 bis $4 \mu$. Mit l.-m. Beobachtungen übereinstimmend, sind die Proplastiden anfangs nur 0,6 bis $0,8 \mu$ breit und zeigen häufig hantelförmige Einschnürungen (vgl. Abb. 2). In den etwa $1 \mathrm{~cm}$ langen Blättchen sind die Proplastiden bis zu 1,2 $\mu$ breit und im Schnitt mehr oval (Abb. 4 bis Abb. 6). Das Primärgranum liegt ungefähr in der Größenordnung von 0,3 bis $0,8 \mu$. In den jüngeren Proplastiden scheint es mehr abgeplattet linsenförmig zu sein, im älteren Proplastid erscheint es mehr kugelig. Bei den GröBenangaben ist jedoch immer zu berücksichtigen, daß im ultradünnen Schnitt je nach Richtung und Ebene des Schnittbildes beträchtliche Differenzen auftreten.

In der gleichen Größenordnung liegt aber auch der von $\mathrm{Heitz}{ }^{12}$ e.-m. untersuchte ,junge Chloroplast" und dessen „Granum“. Nachdem in den hier vorgenommenen umfangreichen Untersuchungen in keinem Falle kleinere Proplastiden aufzufinden waren, ebensowenig solche, denen das Primärgranum offensichtlich fehlte, muß gefolgert werden, daß die von $\mathrm{He}$ itz untersuchten jungen Chloroplasten im Sinne der Literatur „Proplastiden“ — also primäre Entwicklungsstadien von Chloroplasten - sind. Im Interesse der weiteren Erforschung der Plastiden und ihrer Entwicklung wäre es wünschenswert, in $\mathrm{Zu}$ kunft der von $\mathrm{S}$ tr u g g e r ${ }^{2.3}$ erstmalig eingeführten Nomenklatur zu folgen, nachdem nunmehr eine Übereinstimmung zwischen 1.- und e.-m. Befunden besteht. Aus den gleichen Gründen muß auch die von Le y o ${ }^{15}$ allein auf Grund e.-m. Eigenschaften gewählte Terminus „,dense core“ für das Primärgranum der Proplastiden abgelehnt werden. L e y o n bestätigt selber, daß dieses „dense core“ mit dem Primärgranum identisch sei (,i.e. the primary granum according to S trug g e r "). Wenn der von S trug ge r gewählten Bezeichnung „Primärgranum“ der Vorzug gegeben wird, so handelt es sich nicht nur um die Priorität, sondern vielmehr darum, daß nicht nur der e.-m. Aspekt, sondern auch noch andere 1.-m. erfaßbare Eigenschaften im fixierten und lebenden $\mathrm{Zu}$ stand zur Kennzeichnung dieses persistierenden Bestandteils der Proplastiden herangezogen werden.

Bei der e.-m. Analyse der Proplastiden von Chlorophytum comosum haben sich außerdem keine Anhaltspunkte für die allgemeine Richtigkeit der von $\mathrm{M}$ üh lethal e r ${ }^{10}$ vertretenen Ansicht ergeben. Bei Aspidistra elatior wurden Proplastiden als primäre Entwicklungstadien von Chloroplasten aufgefunden, welche nur aus einem Primärgranum bestanden. Weiterhin war es nach $\mathrm{M} u ̈ h l e t h a l e r$ schwer, diese Proplastiden von den im e.-m. Bild ähnlich aussehenden Sphärosomen zu unterscheiden. Abgesehen davon, daß diese Feststellung in absolutem Widerspruch zu entsprechenden l.-m. Beobachtungen steht ${ }^{2-4}$, ist nach den hier vorliegenden e.-m. Befunden an Chlorophytum zu vermuten, daß die Auffassung Mühlethalers auf e.-m. Bildern ungünstiger Schnittrichtung beruht (vgl. Abb. $3 \mathrm{a}$ und b). Bei nahe verwandten Pflanzen ist das Primärgranum nach $\mathrm{S}$ tr u g g e r ${ }^{2-4}$ sehr groß und liegt häufig terminal am Ende des Proplastids, wobei das Stroma an dieser Stelle nur als dünne Schicht ausgebildet ist.

Ungeklärt erscheint nun lediglich die Frage nach der e.-m. nachweisbaren Feinstruktur des Primärgranums im Bereich des Vegetationskegels und in den sich entwickelnden Blättern. Beim Vergleich der Abb. 1 bis 6 ergibt sich, daß das Primärgranum zwar überall vorhanden ist, daß aber der e.-m. Aspekt scheinbar nicht in allen Bildern gleich ist. Man könnte daraus folgern, daß sich die "Kristallgitterstruktur“ erst allmählich mit dem Heranwachsen der Blätter ausbildet, denn in den Proplastiden der älteren, etwa $1 \mathrm{~cm}$ langen Blättchen ist das Primärgranum deutlich geordnet, in den Proplastiden des Vegetationskegels und der jüngsten Blattanlagen zeigt das Primärgranum nur undeutlich Tubuli ohne besonderen Grad einer Ordnung. Ähnliche Bilder zeigt auch Le yon ${ }^{13.15}$. Dieser Schluß hätte aber zur Voraussetzung, daß der Erhaltungszustand der fixierten Protoplasten in dem untersuchten Material gleich ist. Diese Voraussetzung liegt nicht vor. Schon bei l.-m. Untersuchungen dieses Objektes (vgl. l. c. ${ }^{2-4}$ ) hat es sich herausgestellt, daß sich der VegetationskegelBereich außerordentlich schwer fixieren läßt, bei Blättchen treten diese Schwierigkeiten nicht auf. Durch die e.-m. Analyse lassen sich diese Erfahrungen be-

${ }_{15}$ H. Le y o n, Exp. Cell Res. 7, 265 [1954]. 
stätigen. Im Vegetationskegel-Bereich sind häufig Auftreibungen zu beobachten, das Gewebe zerreißt leicht, und das Cytoplasma läßt die feingranuläre Struktur vermissen, wie sie bei anderen Objekten aufgefunden wurde (z. B. beim Vegetationskegel von Helianthus tuberosus ${ }^{1}$ ). Osmiumsäure dringt beim Fixationsvorgang wahrscheinlich schlecht in die kompakten embryonalen Gewebe ein. Die nicht optimale Schneidefähigkeit läßt darauf schließen, daß dementsprechend auch die Durchdringung mit dem Methacrylat-Gemisch nicht ganz ausreichend sein kann. Z.Z. laufen Versuchsreihen mit dem Ziel, Fixationsund Einbettungsmethoden zu finden, welche auch die e.-m. Untersuchung derartig schwer fixierbarer pflanzlicher Meristeme gestatten.

Bei der Beurteilung der Innenstruktur des Primärgranums muß demnach berücksichtigt werden, daß im Vegetationskegel-Bereich mit sekundären Veränderungen der strukturellen Ordnung stärker zu rechnen ist als in etwas älteren Blättchen. Wenn nun trotz dieser Einschränkung beim Vergleich der Abb. 1 bis 6 übereinstimmend immer die gleichartigen osmiophilen Systeme in Erscheinung treten, wie sie bei stärkerer Vergrößerung noch einmal in Abb. 7 gezeigt werden, liegt die Vermutung nahe, daß in vivo das Primärgranum in dem hier untersuchten Material einen gleichartigen Aufbau haben dürfte. Bei der Labilität meristematischer Protoplasten und der unzureichenden Fixation von Meristemen läßt sich aber die Gleichartigkeit im Strukturaufbau e.-m. noch nicht voll befriedigend nachweisen.

\section{E.-m. Beobachtungen zur Frage der „Kristall- gitterstruktur“ im Primärgranum}

Davon ausgehend, daß ein Primärgranum in allen Proplastiden des Vegetationskegels und der jüngeren Blätter persistierend nachweisbar ist, aber in den Blättchen von 1 bis $3 \mathrm{~cm}$ Länge den besten Erhaltungszustand aufweist, ist dieses Material zur näheren Analyse der Feinstruktur verwendet worden. Nach $\mathrm{Heitz}{ }^{12}$ stellt das Primärgranum (Granum junger Chloroplasten im Sinne von $\mathrm{Heitz}$ ) einen Kristall dar: „Das Granum junger Chloroplasten besteht demnach nicht aus parallel liegenden Lamellen, sondern aus einem oder mehreren aneinander sitzenden Kristallen, deren Kristallgitter e.-m. an Dünn-

16 E. R a bi novitch u. Mitarb., Z. Phys. 133, 261 [1952]; S. T a k a s him a, Nature [London] 169, 182 [1952]; F. A. R odrigo, Biochim. biophysica Acta [Amsterdam] 10, 342 [1953]; D. R. A nd e r s on u. Mitarb., Biochim. biophysica Acta [Amsterdam] 15, 298 schnitten nachweisbar sind." Nähere Angaben über Größe der „Gitterpunkte“ und ihren Abstand werden nicht gemacht. An anderer Stelle wird ausgeführt: „Bei Erreichung einer bestimmten Konzentration des im Stroma gebildeten Chlorophylls kommt es infolge von Kristallisation zur Bildung der Grana, der Bildung von Stärke aus Zucker vergleichbar." Dieser Auffassung über die Natur des Primärgranums muß jedoch aus verschiedenen Gründen widersprochen werden:

1. Wie vorher gezeigt worden ist, entsteht der von Heitz beschriebene, stark elektronenstreuende Komplex - das Primärgranum - nicht erst im Zuge der Ontogenese zum Chloroplasten, sondern er ist bereits primär vorhanden. Diese Befunde stehen in absoluter Übereinstimmung zu l.-m. Beobachtungen an fixiertem Material ${ }^{1}$ und zu fluoreszenzmikroskopischen Untersuchungen über die Verteilung des Chlorophylls ${ }^{5}$.

2. Nach den Ergebnissen biochemischer Untersuchungen ${ }^{16}$ liegen Hinweise dafür vor, daß Chlorophyll in vivo an Eiweißkörper gebunden ist und demnach als Chromoproteidkomplex vorliegen dürfte. Es ist gelungen, diesen Komplex $\mathrm{zu}$ isolieren und zur Kristallisation zu bringen. Dann ist die typische Chlorophyllfluoreszenz festzustellen, leider liegen aber nähere Angaben über Gitterkonstante und andere physikalische Daten nicht vor. Ob das e.-m. festgestellte "Gitter" in irgendeiner Weise mit dem in vitro zur Kristallisation gebrachten Chromoproteid aus Chloroplasten vergleichbar ist, läßt sich nicht entscheiden, dürfte aber unwahrscheinlich sein. Die Möglichkeit, daß die beobachtete „Gitterstruktur“ das Kristallisationsprodukt eines makromolekularen Chromoproteids sein könnte, ist von $\mathrm{He}$ it z nicht in Erwägung gezogen worden.

J a c obs und Mitarbeiter ${ }^{17}$ haben Chlorophyll a in vitro zur Kristallisation gebracht, wobei ,triangular sheets and stocked bimolecular layers" beobachtet wurden. Auf Grund einer röntgenographischen Analyse wird für $D_{\text {HKl }}$ der Wert von 22,0 $\AA, 14,6 \AA$ und $7,5 \AA$ angegeben. Im Primärgranum ist ein „Kristall“ dieser Eigenschaften nicht nachweisbar, der Abstand der einzelnen „Gitterpunkte“ beträgt ein Vielfaches davon.

3. Gegen die Annahme, daß im Primärgranum ein Chlorophyllkristall vorliegt, sprechen auch die fol-

[1954]; Y. Chiba, Arch. Biochem. Biophysics 54, 83 [1955].

17 E. E. J a c obs u. Mitarb., J. chem. Physics 21, 2246 [1953]. 

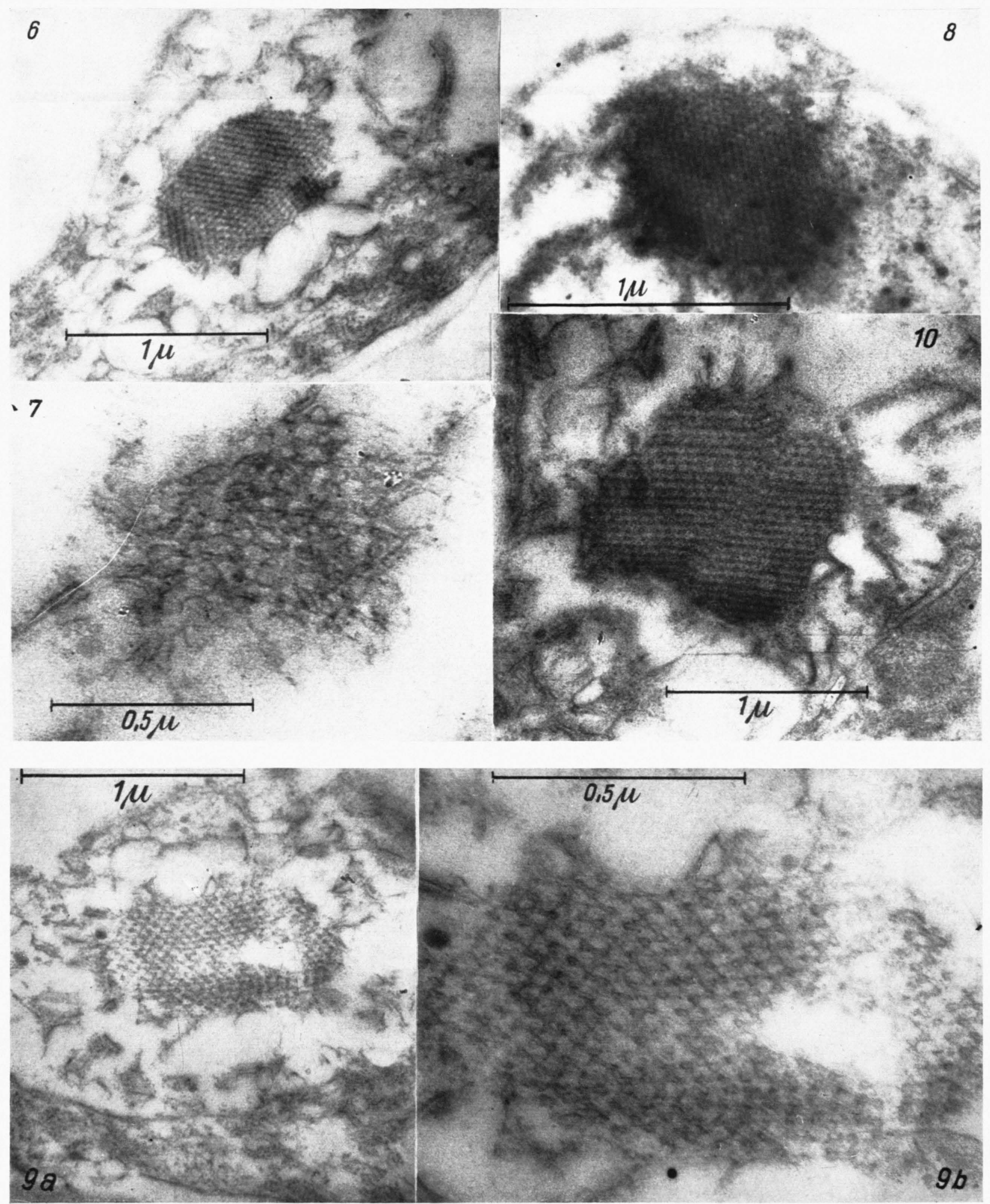

Abb. 6. Ideal getroffenes Proplastid aus einem etwa $1 \mathrm{~cm}$ langen Blatt, bei dem das Primärgranum bereits die typipische „kristallgitter-artige“ Struktur zeigt (Pr.-Vergr.: $16500)$.

Abb. 7. Detail aus dem Primärgranum eines Proplastids im Vegetationskegel-Bereich bei nicht optimalem Erhaltungszustand (Pr.-Vergr.: 44000 ).

Abb. 8. Nach 23 Tagen Extraktion mit abs. Alkohol zeigt das Primärgranum aus einem $1 \mathrm{~cm}$ langen Blatt noch die typische Struktur (Pr.-Vergr.: 13 600).
Abb. 9 a. Das Primärgranum eines Proplastids aus einem $1 \mathrm{~cm}$ langen Blatt bei 16500 -facher Primärvergrößerung. Die „Gitterpunkte“ werden nur undeutlich aufgelöst.

Abb. 9 b. Ausschnitt aus dem gleichen Primärgranum bei 44000 -facher Primärvergrößerung. Es sind sowohl die Elementareinheiten als auch die fädigen Verbindungen zu erkennen.

Abb. 10. Primärgranum mit deutlich sichtbarer Lamellenstruktur bei idealer Schnittrichtung (Pr.-Vergr.: 16 500). 

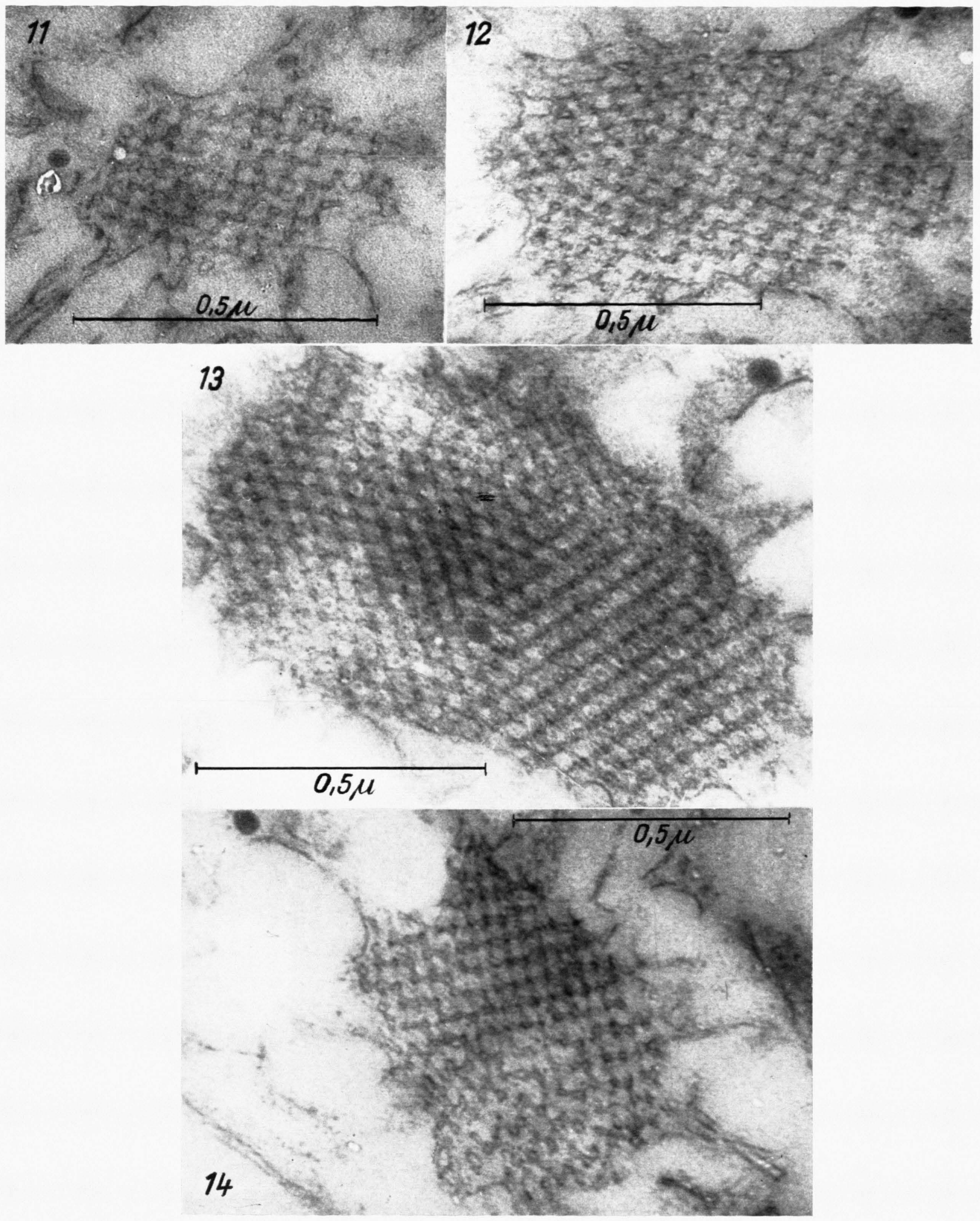

Abb. 11. „Parkettmuster“-artige Verbindung zwischen den einzelnen Elementareinheiten im Primärgranum (Pr.-Vergr.: 44000$).$

Abb. 12. Primärgranum eines Proplastids bei 44000 -facher Primärvergrößerung, wobei die Ordnung der einzelnen Elementareinheiten deutlich wird.
Abb. 13. Primärgranum eines Proplastids bei 44 000-facher Primärvergrößerung mit nicht näher deutbarer Ordnung.

Abb. 14. Im unteren Teil des Primärgranums ist eine Störung der Ordnung eingetreten, welche wahrscheinlich auf eine unzureichende Fixation zurückzuführen ist (Pr.-Vergr.: 44 000). 
G. Melchers, Die Beteiligung der endonomen Tagesrhythmik am Zustandekommen der photoperiodischen Reaktion der Kurztagpflanze Kalanchoë blossfeldiana (S. 544)

1

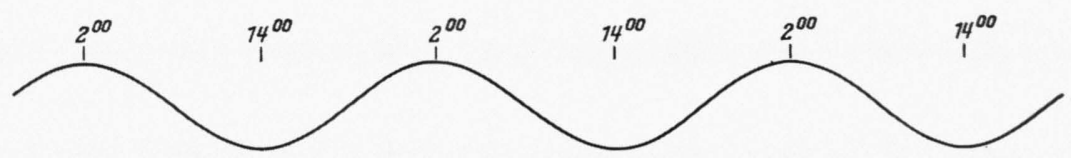

2
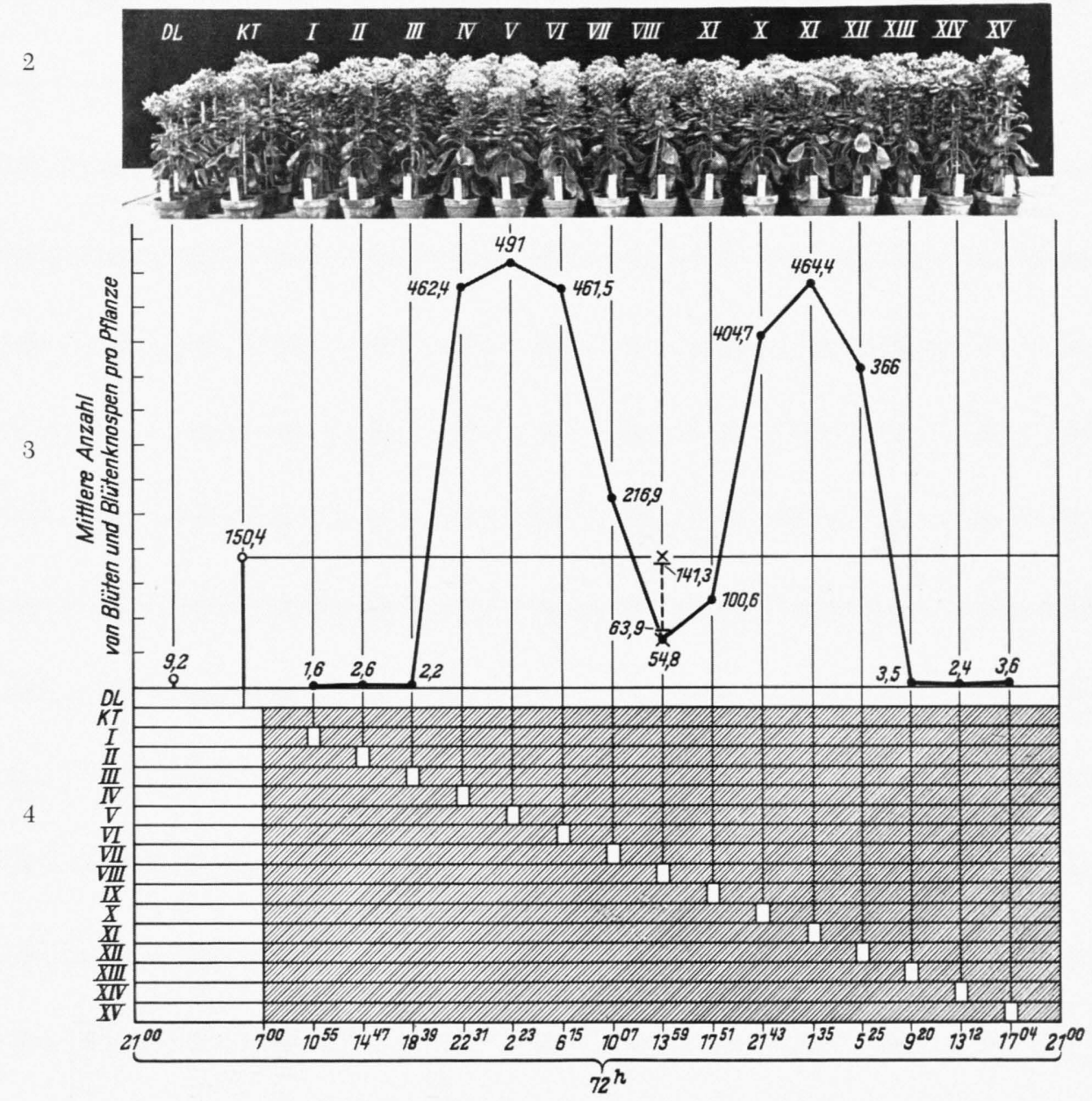

Abb. 1. Von oben nach unten: 1. Verlauf der „endonomen Tagesrhythmik" unter der Annahme, daß in der Mitte der Hauptlichtzeit (200 Uhr) das erste Maximum der photophilen Phase liegt. 2. Zustand der Versuchspflanzen (Gruppe I-XV) und der Dauerlicht-(DL) und Kurztag-(KT)-Kontrollen zur Zeit der Auswertung. 3. Die mittlere Anzahl von Blüten und Blütenknospen bei den Kontrollen und Versuchsgruppen am Ende des Versuchs (bei Gruppe VIII ist + + + die höchstzulässige Zufallsdifferenz zwischen dem Mittelwert und der KT-Kontrollgruppen eingetragen). 4. Verteilung von Licht 0 und Dunkelheit 2 in einem der 9 im Versuch durchgeführten $72 \mathrm{~h}$ Tagen. $2100-700$ Uhr des ersten Tages $=$ Hauptlichtzeit. $10^{55}, 14^{47}$ Uhr usw. = Mitte der einstündigen Zusatzbeleuchtung der Versuchsgruppen.

Photo: Ursula Engelmann; Zeichnung: Erich Freiberg. 
genden e.-m. Untersuchungen. Wie aus Abb. 8 ersichtlich, läßt sich das Gitter noch in absolut gleicher Weise e.-m. darstellen, wenn im Zuge der Einbettung die in üblicher Weise mit Osmiumsäure fixierten Objekte 23 Tage in abs. Alkohol liegen. In dieser Zeit erfolgt zweifellos eine Extraktion alkohol-löslicher, osmiophiler Substanzen, denn das Medium nimmt auch bei mehrfachem Wechsel eine schwarze bzw. braune Farbe an. Die Fixation mit Osmiumsäure vermag eine solche Extraktion nicht zu verhindern, was mit früheren Erfahrungen bei Allium-Epidermen ${ }^{18}$ übereinstimmt. Der Charakter der extrahierten Stoffe läßt sich nur vermuten. Wahrscheinlich sind es unter anderem Fette bzw. Lipoide, denn bei Allium-Epidermen wurde die Sudanfärbung schließlich negativ. Wenn nun hier noch nach 23 Tagen Alkoholextraktion der gleiche gitterartige Aspekt ohne irgendwelche Verminderung der Intensität der Elektronenstreuung festzustellen ist, kann dieser Komplex nicht aus Lipoiden und ähnlichem bestehen, wie es $\mathrm{He} \mathrm{itz}^{12}$ postuliert. Es sind vielmehr Stoffe beteiligt, die gegenüber abs. Alkohol unempfindlich sind und dazu Osmiumsäure zu reduzieren vermögen. Dabei ist an Proteide und nach, den cytochemischen Befun-

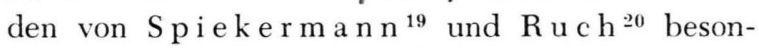
ders an Nucleoproteide zu denken. Das e.-m. Bild gibt nach Osmiumsäure-Fixation keine Auskunft über das Vorhandensein von Nucleinsäuren ${ }^{21}$, denn diese vermögen in vitro im Modellversuch Osmiumsäure nicht zu reduzieren. Daß sie aber im Primärgranum der Proplastiden vorhanden sind, haben unabhängig voneinander und mit verschiedenen Methoden Spiek e r m an $n$ und $\mathrm{R} \mathrm{u} \mathrm{ch} \mathrm{gezeigt}{ }^{19,}{ }^{20}$. Im Zusammenhang mit enzymatischer Verdauung durch Nucleasen ist dies neuerdings sowohl mit Färbemethoden als auch durch UV-Mikrospektrographie erwiesen worden. Wenn auch Nucleinsäuren bei den üblichen Methoden e.-m. nicht nachgewiesen werden können, so sind doch in jedem Falle die Eiweißkomponenten zu einer Reduktion von Osmiumsäure befähigt und „färben " sich e.-m. an. Das Primärgranum muß danach einen Komplex darstellen, welcher Chlorophyll, Nucleinsäuren, Lipoide und Proteine enthält.

4. Bei dieser vermutlichen stofflichen Zusammensetzung des Primärgranums stellt das Primärgranum strukturell einen „Chromo-Nucleo-Proteid-Komplex“ dar, und es ist unwahrscheinlich, daß er als ,Kristall“ vorliegt. Daraus ergibt sich die Notwendigkeit, sich

18 E. S. Perner, Biol. Zbl. 71, 43 [1952]; E. S. P e rner, Protoplasma 42, 457 [1953].

19 R. S p i e k e rm a n n, Inaug.-Diss., Münster (Westf.) 1956. durch die volle Ausnutzung der hohen Auflösung des Elektronenmikroskops (high resolution im Sinne anderer Autoren) einen näheren Einblick in die Strukturordnung des Primärgranums zu verschaffen. An besonders geeigneten ultradünnen Schnitten gelang es, eine stärkere Primärvergrößerung (bis zu 44000 fach) anzuwenden, als es bislang der Fall war. $\mathrm{H}$ e i t z gibt eine Primärvergrößerung von 4050-fach an, in diesen Untersuchungen wurde meist mit 8800und 16500 -facher Primärvergrößerung gearbeitet. Nach dem Ergebnis. dieser Untersuchungen besitzt das Primärgranum einen wesentlich komplizierteren $\mathrm{Bau}$, als es bislang vermutet wurde, und von einem „Kristall“ kann keine Rede mehr sein.

Nach Heitz ${ }^{12}$ zeigt ,,jede Reihe eine Differenzierung in dunklere, kreisrunde Flächen, die in sehr regelmäßigen Abständen mit etwas helleren, durch parallele Linien begrenzte Flächen miteinander wechseln“. Dieser Aspekt kann voll bestätigt werden, wenn lediglich eine schwächere Primärvergrößerung verwendet wird (vgl. Abb. 6 und Abb. 9 a), und er mag $\mathrm{H}$ e it $\mathrm{z}$ veranlaßt haben, in dem Primärgranum einen Kristall zu sehen.

Bei stärkerer Primärvergrößerung lassen sich die „,kreisrunden, dunkleren Flächen“ als angebliche Gitterpunkte des Chlorophyllkristalls weiter auflösen. Hat man bei schwächerer Vergrößerung (Abb. 9 a, 16 500-fache Primärvergrößerung) den Eindruck, daß diese Gitterpunkte einheitlich osmiert sind und annähernd homogen schwarz erscheinen, bietet sich beim gleichen Primärgranum - aber 44000 -facher Primärvergrößerung - in Abb. $9 \mathrm{~b}$ ein anderes Bild. Die annähernd kreisrunden Gitterpunkte zeigen bei einem Durchmesser von etwa $190 \AA$ nur in den äußeren Schichten eine stärkere Elektronenstreuung, im Inneren sind sie dagegen nicht osmiert und erscheinen damit hell. Derartige Bilder zeigten sich übereinstimmend, auch wenn die Fixationsdauer auf 12 Stdn. ausgedehnt wurde, gleichgültig ob sie bei Zimmertemperatur oder $+2^{\circ} \mathrm{C}$ vorgenommen wird. Die angeblichen „Gitterpunkte“ müssen demnach stofflich heterogen aufgebaut sein, was sich aus dem Äquivalentbild der Osmiumniederschläge ergibt. Zur Reduktion von Osmiumsäure sind demnach nur die in den äußeren Schichten dieser „Elementareinheiten“ befindlichen Stoffe befähigt, im Inneren befinden sich vorwiegend solche, die nicht osmiophil sind. Z. Z. laufen Untersuchungen, um mit Hilfe des E.-M. in

20 P. D. R u c h, Vortrag Tg. Dtsch. Bot. Ges. 1956 in Hann.-Münden.

21 G. F. B a h r, Exp. Cell Res. 7, 457 [1954]. 
Verbindung mit cytochemischen Methoden die Natur der einzelnen Komponenten in den Elementareinheiten näher aufzuklären.

An Hand der folgenden e.-m. Bilder des Primärgranums, welches dabei in verschiedenen Richtungen und Ebenen getroffen wurde, ergibt sich, daß diese Elementareinheiten geordnet liegen und wie eine „Perlkette“ auf einem fädigen Strukturelement im Abstand von etwa $450 \AA$ aufgereiht sind. Diese fadenartige Verbindung zwischen den einzelnen Elementareinheiten tritt in den Bildern gelegentlich als eine Doppellinie in Erscheinung und ist in Abb. $9 \mathrm{~b}$ und Abb. 11 besonders deutlich. Nachdem das Primärgranum immer wieder als ein lamellierter Schichtenkörper in Erscheinung tritt, wie es Abb. 10 zeigt, ist anzunehmen, daß sich die perlketten-artigen Fäden mit den Elementareinheiten in bevorzugten Ebenen miteinander vernetzen, so daß der Aspekt eines Flächengitters zustande kommt (vgl. Abb. 9 b, 11, 12 und 13). Aber bereits Abb. 10 zeigt, daß die lamellenartige Ordnung nicht in jedem Falle ideal und ohne Störung ist. So kann manchmal beobachtet werden, daß die Verbindung zwischen den einzelnen Elementareinheiten regelmäßig wechselt, so daß das Primärgranum ein „Parkettmuster“ zeigt (vgl. Abb. 11). In den Schnittbildern sind aber immer wieder auch andere Ordnungsprinzipien festzustellen, wie aus Abb. 12 und Abb. 13 zu ersehen ist. Sie lassen sich z. Z. noch nicht deuten. Abb. 14 zeigt dann ab- schließend, daß auch mit einer sekundären Störung dieser hohen Ordnung im Primärgranum gerechnet werden muß. Während im oberen Teil des Primärgranums alle Elementareinheiten geordnet liegen, herrscht unten im Bild eine weitgehende Desorganisation. Sie kann möglicherweise durch eine ungenügende Fixation bedingt sein.

Die bislang im Schrifttum e.-m. dargestellten makromolekularen Kristalle biogener Herkunft ${ }^{22}$ zeigen niemals einen Aspekt, wie er hier im Primärgranum der Proplastiden aufgefunden wurde. Gegen die von $\mathrm{H}$ e it z vermutete Kristallnatur spricht nicht nur der verschiedenartige Aspekt, sondern auch die Größenordnung. Zur weiteren Aufklärung des Strukturaufbaus im Primärgranum der Proplastiden von Liliifloren wäre es wünschenswert, ein Primärgranum möglichst lückenlos in Einzelschnitten einer Serie zu erfassen. Bei einer Dicke des Primärgranums von ca. $0,5 \mu$ wären dazu aber 50 Schnitte nötig, wenn diese $100 \AA$ dick sind. Entsprechende Versuche sind unternommen worden, haben aber nur bis zu 10 Bildern geführt. Wenn sie noch nicht zur Anfertigung eines Modells ausgereicht haben, zeigt dies die in Wirklichkeit vorliegenden komplizierten Verhältnisse. Hier von einem einfachen bzw. zusammengesetzten Kristall zu sprechen, entspricht in keinem Falle den Tatsachen.

22. A. Frey-Wyss ling, Protoplasmatologia Bd. II, A 2, Springer-Verlag, Wien 1955. 